\title{
Hacia la articulación del derecho estatal e indígena en casos de violencia contra la mujer en la Costa Caribe de Nicaragua ${ }^{1}$
}

\author{
Towards the articulation of state and indigenous law in cases of violence against \\ women in the Caribbean Coast of Nicaragua
}

\author{
Sandra Carolina Rojas Hooker²
}

\section{Resumen}

El presente trabajo ha presentado los aportes de la URACCAN como Universidad Comunitaria Intercultural en el tema de Administración de Justicia Intercultural; se ha utilizado una investigación cualitativa a un nivel descriptivo. La inclusión de esta temática en la Política de Extensión Social Comunitaria de URACCAN, facilitó los espacios de formación, intercambio de experiencia y sesiones de trabajo conjunto entre autoridades del sistema estatal y autoridades ancestrales, entre otros espacios servirá de base para la elaboración de la propuesta que articula el derecho indígena y estatal en los casos de violencia contra la mujer en la Costa Caribe de Nicaragua.

Palabras clave: pluralismo jurídico; administración de justicia; derechos de la mujer.

\section{Abstract}

The work has presented the contributions of the URACCAN as Intercultural Community University in the topic of Intercultural Justice Administration; a qualitative research approach has been used at a descriptive level. The inclusion of this topic in the Community Social Extension Policy of URACCAN facilitated training spaces, exchange of experiences and joint working sessions among authorities of the state system and ancestral authorities, and other spaces. This work will serve as the basis for the preparation of the proposal that articulates the indigenous and state law in cases of violence against women in the Caribbean Coast of Nicaragua.

Key words: Legal Pluralism; Justice Administration; Women's Rights.

\section{Introducción}

El surgimiento de movimientos indígenas en el escenario político latinoamericano en la década de los 70 demandó el reconocimiento, vigencia y reivindicación de un derecho propio que regule su vida como indígenas. Uno de los grandes aspectos a tratar es "el sistema jurídico consuetudinario indígena tradicional" y su relación con el Estado nacional.

\footnotetext{
$\overline{1}$ XIV Congreso Latinoamericano y del Caribe de Extensión Universitaria y Segundo Congreso Centroamericano de Compromiso Social para la vinculación social de la universidad con la sociedad.

2 Máster en Estudios Indígenas. Directora del Instituto de Estudios y Promoción de la Autonomía de la Universidad de las Regiones Autónomas de la Costa Caribe Nicaragüense.Correo: sandra.rojas@uraccan.edu.ni ORCID https://orcid.org/0000-0002-7467-2366
} 
Para entender esta relación utilizamos el concepto de pluralismo jurídico para explicar que el derecho está lejos de ser un producto exclusivo de determinadas sociedades. Dentro de los confines del Estado puede haber diversas manifestaciones de derecho. Implica la aceptación de varios órdenes que pueden convivir en un mismo espacio y tiempo, negando la exclusividad del derecho estatal en la producción de normas jurídicas. La premisa de este concepto es que el Estado no es la única fuente de normas y prácticas legales, sino que coexiste con muchos otros espacios donde se generan normas y se ejerce control social.

En aras de contribuir al reconocimiento y ejercicio efectivo de los derechos humanos y autonómicos de los pueblos de la Costa Caribe de Nicaragua la URACCAN en el 2005 define como una de las temáticas de la extensión social comunitaria la Administración de Justicia Intercultural con miras a un reconocimiento real y efectivo de la pluralidad legal existente en el país.

\section{Materiales y métodos}

El estudio es fundamentalmente de tipo descriptivo, desarrollado con metodologías cualitativas y con el soporte de la información cuantitativa disponible. Las principales técnicas de investigación utilizadas fueron las sesiones de reflexión, los grupos focales y la revisión documental.

Las sesiones de reflexión con abogadas costeñas y lideresas comunitarias para poder analizar los avances en términos de implementación de la justicia regional y revisar los abordajes desde las comunidades. También para conocer las dificultades que se encuentran en el proceso, las alianzas y coordinaciones de las organizaciones de mujeres y las autoridades comunitarias y del poder.

Los grupos focales se realizaron en las Regiones Autónomas de la Costa Caribe Nicaragüense Norte y Sur (RACCN y RACCS), en Bluefields, las comunidades de Orinoco y la Desembocadura de Río Grande y en Bilwi, Bonanza y Waspam.

La revisión documental fue necesaria para conocer los avances que se han realizado en los espacios gubernamentales, ONGs, así mismos procesos que se han llevado a cabo en la universidad URACCAN.

Para estas sesiones, metodológicamente se contó con una guía de preguntas semi-estructuradas que fueron diseñadas en base a las realidades de las comunidades y bajo las líneas temáticas a abordar según lo previsto en esta propuesta.

\section{Hacia la articulación del derecho indígena y estatal en los casos de violencia contra la mujer en la Costa Caribe nicaragüense}

La administración de justicia en comunidades indígenas y afrodescendientes parte de la concepción fundamental de restablecer el orden en la comunidad y la rehabilitación del individuo como tal. Persigue la búsqueda del bien común. Las sanciones aplicadas por las autoridades indígenas se basan en la justicia restaurativa. Ello no sólo se limita a las regulaciones referentes a los asuntos contenciosos (solución de conflictos, aplicación de penas por violación de las reglas), sino que incluye regulaciones relativas a la propiedad comunal, y a asuntos de carácter civil y de regulación de las autoridades, en muchos casos difíciles de separar del conjunto de las prácticas culturales cotidianas de estos grupos.

Los artículos 5, 89, 90, 180 y 181 de la Constitución Política de Nicaragua reconocen la existencia de los pueblos originarios y afrodescendientes, que gozan de los derechos, deberes y garantías consignados en la Constitución y en especial, los de mantener y desarrollar su identidad y cultura, tener sus propias formas de organización social y administrar sus asuntos locales; así como mantener las formas comunales de propiedad de sus tierras y el goce, uso y disfrute, todo de conformidad con la Ley. Para las comunidades de la Costa Caribe se establece el régimen de autonomía. 
Conforme lo anterior, el artículo 18 de la Ley 28 establece: "La administración de la justicia en las Regiones Autónomas se regirá por regulaciones especiales que reflejan las particularidades culturales propias de las comunidades de la Costa Caribe de Nicaragua". En tanto, el artículo 33, del reglamento de la Ley 28 dice: "Las autoridades comunales administran justicia dentro de las comunidades y entre los comunitarios, de conformidad con sus costumbres y tradiciones." Estos artículos reconocen la potestad legal de las autoridades comunales de administrar justicia como parte de la administración de los asuntos locales.

En este tejido legal, los esfuerzos de articulación entre el sistema estatal y propio promueven el respeto y reconocimiento de procedimientos e instituciones que favorecen las relaciones multiétnicas e interculturales dentro del marco de justicia social. A la vez, alienta la vinculación armoniosa y constructivista del Derecho estatal y el Derecho de los pueblos indígenas y afrodescendientes de la Costa Caribe nicaragüense.

El Estado de Nicaragua al ser signatario de instrumentos internacionales que protegen y promueven los derechos de la mujer promulga en el año 2012 la Ley 779, Ley Integral contra la Violencia hacia la mujer y de Reforma al Código Penal con el propósito de proteger los derechos humanos de las mujeres y garantizarles una vida libre de violencia que favorezca su desarrollo y bienestar conforme los principios de igualdad y no discriminación.

Sumado a esto, se reconoce la Jurisdicción Indígena y Afrodescendiente mediante el cual las autoridades de estos pueblos tienen la potestad de resolver los conflictos entre los integrantes de sus comunidades, conforme el Derecho Consuetudinario en los delitos y faltas cuya pena no exceda de cinco años de prisión.

El respeto y el reconocimiento a los sistemas de justicia propia, debe de ir en consonancia con lo estatuido en las diferentes normativas jurídicas referente al respeto a las garantías individuales, los derechos humanos y, en particular, la dignidad e integridad de las mujeres y en especial el derecho a una vida libre de violencia.

La violencia hacia las mujeres indígenas rebasa la visión tradicional de comprender la problemática únicamente desde la perspectiva de los derechos individuales, habrá que hacer un esfuerzo por representar, por un lado, la violencia de género producida dentro de los propios pueblos y que reflejan la desigualdad entre los géneros. Por otro lado, la violencia estructural que afecta al colectivo ligada a la violencia ejercida por el Estado en su contra o bien, de otros pueblos hacia ellos. Estos dos niveles de análisis cruzan nuestra reflexión sobre la afectación de los derechos colectivos e individuales de las mujeres indígenas.

Dentro de este tejido social, se observa que las mujeres indígenas están recurriendo en búsqueda de soluciones para detener la violencia en sus relaciones de pareja. Las mujeres acuden a la administración de justicia para la atención de sus quejas, buscando como es frecuente una medida de reparación. Cuando las mujeres se mueven para obtener apoyo dos espacios jurídicos interactúan: la comunidad y el Estado, de manera que, en el camino recorrido, las mujeres pueden encontrar una diversidad de dispositivos en la procuración de justicia. No obstante, las atenciones brindadas por estas instancias difieren.

El Modelo Atención Integral (MAI) pretende garantizar la coordinación interinstitucional con el fin de: "proporcionar a las mujeres, niñas y niños, víctimas de violencia intrafamiliar y sexual, servicios que garanticen su protección, seguridad personal y el acceso a la justicia, evitando la victimización secundaria. Busca, mejorar la atención que brinda el Estado en la ruta crítica a las víctimas de violencia desde que inicia hasta que culmina los procesos legales garantizando el enfoque de derechos humanos, género, generacional e intercultural en la búsqueda de consensos para evitar duplicidades y exclusiones en la atención.

En esta ruta de acceso, con las mujeres afectadas se entreteje una red de apoyo en la cuales interactúan autoridades ancestrales, caso particular de los Jueces comunales llamados wihtas, policías voluntarios, movimiento de mujeres y líderes religiosos que acompañan en el tema de promoción y protección de los derechos humanos. En este caminar un conjunto de actores como el jefe de sector, Comisaria de la Mujer ahora Auxilio Judicial, Ministerio Público a través de la Fiscal de Género, Defensoría Pública y Autoridades 
Judiciales son los encargados de conocer y resolver el caso que se les presenta con miras a que las mujeres puedan integrarse nuevamente a la sociedad y ejercer sus labores cotidianas.

Las organizaciones de mujeres, han desempeñado un rol fundamental, siendo la red de apoyo al brindarles atención y acompañamiento a las autoridades policiales y judiciales durante todo el proceso que se ventila ante esta instancia. Sin dejar a un lado el apoyo espiritual que reciben de las autoridades religiosas, familiares, y de mujeres sobrevivientes ayuda a tener fuerza, valor y a seguir adelante, en palabras de una entrevistada.

Este apoyo es sin duda indispensable no sólo durante el proceso de búsqueda de la justicia, sino después, durante el proceso de recuperación. A como plantea la Lagarde (2009), "qué sería de las mujeres sin el aliento y el apoyo en situaciones de crisis que son tantas".

El estudio denominado "Hablando de Justicia", aproximaciones sobre el acceso de las mujeres a la justicia en el contexto de la aplicación de la ley 779 en Nicaragua, realizado por el CEIMM - URACCAN permitió contar con información actualizada sobre la presente temática que sirvió para replantear algunos aspectos claves para el abordaje de los derechos de la mujer para garantizar el acceso a la justicia en un contexto multiétnico e intercultural.

Dando continuidad a los procesos de articulación entre el derecho positivo y derecho propio en el abordaje de los derechos de las mujeres, realizamos dos sesiones de reflexión "repensando la administración de justicia intercultural, para el verdadero ejercicio de los derechos de las mujeres y de la niñez en la Costa Caribe nicaragüense" con la participación de abogadas de las dos Regiones Autónomas, mujeres de organizaciones afines a la temática y líderes que han impulsado procesos desde las comunidades y mujeres en espacios estratégicos relacionados al proceso como el Poder Judicial y la Procuraduría de la Mujer.

Estas sesiones se centran en analizar las buenas prácticas de los sistemas de justicia en los delitos de violencia hacia las mujeres, que contribuyan a la construcción de una guía de armonización en los contextos multiétnicos mediante el análisis, reflexión y abordaje de la misma partiendo de la comunidad que nos ayuda a tener un enfoque integral al proceso en el marco del respeto a los Derechos Humanos y Autonómicos.

También se plantea el seguimiento a la propuesta de modelo de justicia para la Costa Caribe nicaragüense impulsado por la Comisión de Justicia Comunal Costeña, aprobado por los Consejos Regionales y presentado a la Corte Suprema de Justicia en septiembre del 2009 documento que vendría a enriquecer ambos sistemas de derechos y acercarlo más a la realidad de los costeños, incentivando las formas de administración justicia de los pueblos indígenas y afrodescendientes para así reforzar también su identidad y su organización para que puedan entablar relaciones en un nivel de igualdad y de respeto recíproco.

Analizamos las dificultades que se encuentran en el proceso, las alianzas y coordinaciones de las organizaciones de mujeres y las autoridades comunitarias y del poder judicial, indagando qué nuevas iniciativas o propuestas se deben de considerar para contar con un proceso más inclusivo e integral visto desde la realidad de las mujeres. Un aspecto preponderante es la inclusión de wihtas, líderes religiosos y organizaciones de mujeres en el Modelo de Atención Integral a fin que responda a las realidades de la Costa Caribe nicaragüense, tomando en cuenta la existencia de autoridades ancestrales que administran justicia para poder rescatar las buenas prácticas e incidir para ir erradicando aquellas que violentan los derechos de las mujeres. 


\section{Conclusiones}

La creación de espacios de concertación con los actores del sistema judicial estatal, las universidades costeñas y otros actores sociales se da en un ambiente de respeto y de interacción cultural, donde el conocimiento mutuo y el diálogo son elementos esenciales para la creación y recreación de conocimientos, saberes y prácticas del sistema jurídico de los pueblos indígenas y afrodescendientes.

Constituye un instrumento de avanzada en un contexto multiétnico e intercultural tomando como referencia el reconocimiento constitucional y normativo del sistema jurídico indígena y afrodescendiente que promoverá un cambio significativo en la función judicial mediante el establecimiento de pautas para la coordinación y articulación entre autoridades ancestrales y estatales en la atención a personas víctimas de violencia intrafamiliar, con miras a restaurar la paz y la armonía en la sociedad.

\section{Lista de referencias}

Gaceta, Diario Oficial (1987). Estatuto de Autonomía de las Regiones Autónomas de la Costa Atlántica de Nicaragua, Ley No. 28, Managua, Nicaragua.

Gaceta, Diario Oficial (2007). Código Penal de Nicaragua, Ley No.641, Managua, Nicaragua.

Gaceta, Diario Oficial (2012). Ley Integral de Violencia hacia las mujeres y de reformas a la Ley No 641. Código Penal, Managua, Nicaragua.

Gaceta, Diario Oficial (2014). Ley de Reforma Parcial a la Constitución Política de la Republica de Nicaragua, Ley No. 854, Managua, Nicaragua.

Narváez Campbell Joel, Rojas Hooker, Sandra, et al (2008). Propuesta: Modelo para la administración de justicia de la Costa Caribe de Nicaragua que armoniza la jurisdicción indígena y afro descendientes con la jurisdicción estatal. No publicado.

URACCAN (2005). Política de extensión social y comunitaria, Managua, Nicaragua.

URACCAN (2012). Política y agenda de investigación y extensión, Managua, Nicaragua.

URACCAN (2016). "Hablando de Justicia", aproximaciones sobre el acceso de las mujeres a la justicia en el contexto de la aplicación de la ley 779 en Nicaragua. Managua, Nicaragua. No publicado 